\title{
Herpetomonas trimorpha sp. nov. (Trypanosomatidae, Kinetoplastida), a parasite of the biting midge Culicoides truncorum (Ceratopogonidae, Diptera)
}

Correspondence

Lenka Zídková

murfar@seznam.cz
Lenka Zídková, ${ }^{1}$ Ivan Cepicka, ${ }^{2}$ Jan Votýpka ${ }^{1}$ and Milena Svobodová ${ }^{1}$

\author{
'Department of Parasitology, Faculty of Science, Charles University in Prague, Vinicna 7, Prague \\ 128 44, Czech Republic \\ ${ }^{2}$ Department of Zoology, Faculty of Science, Charles University in Prague, Vinicna 7, Prague \\ 128 44, Czech Republic
}

\section{INTRODUCTION}

The genus Herpetomonas comprises monoxenous trypanosomatids, which mainly parasitize the digestive tract of muscid Diptera (Wallace, 1966; Molyneux, 1977; McGhee \& Cosgrove, 1980; Podlipaev, 1990). However, herpetomonads have also been isolated from plants and even mammals (Morsy et al., 1988; Catarino et al., 2001; Fiorini et al., 2001; Podlipaev et al., 2004a; Marín et al., 2007). The classification of the genus Herpetomonas is problematic and, similar to most other monoxenous trypanosomatid genera, the genus is polyphyletic (Camargo et al., 1992; Teixeira et al., 1997; Hollar et al., 1998; Hughes \& Piontkivska, 2003; Podlipaev et al., 2004a). Therefore, a major taxonomic revision of 'lower' trypanosomatids is needed (see Bulat et al., 1999; Podlipaev, 2000, 2001; Maslov et al., 2001; Merzlyak et al., 2001; Momen, 2001; Yurchenko et al., 2008, 2009).

Over 30 herpetomonad species have been described at the time of writing (McGhee \& Cosgrove, 1980; Podlipaev, 1990). Various authors have used different approaches for

Abbreviations: gGAPDH, glycosomal glyceraldehyde phosphate dehydrogenase; RAPD, random amplified polymorphic DNA.

The GenBank/EMBL/DDBJ accession numbers for the SSU rRNA and gGAPDH gene sequences of $H$. trimorpha sp. nov. are EU179326 and FJ850241, respectively. The accession number for the gGAPDH gene sequence of $H$. ztiplika is FJ850242.

Random amplified polymorphic DNA amplification patterns of isolate CER 9 and related isolates are available with the online version of this paper. the description of new species (Nunes et al., 1994; Teixeira et al., 1997; Maslov et al., 2001; Santos et al., 2005). Morphology-based taxonomy has been criticized by some authors, who argued that most trypanosomatids are morphologically too variable to possess reliable diagnostic characters (Wallace et al., 1983; Batistoti et al., 2001; Merzlyak et al., 2001; Podlipaev, 2001; Yurchenko et al., 2008, 2009). Indeed, the classical diagnostic character, i.e. the presence of promastigotes and opisthomastigotes, is no longer considered to be sufficient for assigning a trypanosomatid to the genus Herpetomonas (Camargo et al., 1992; Podlipaev et al., 2004b). On the other hand, some trypanosomatids were described solely on the basis of their host specificity, following the 'one host - one parasite' paradigm. However, this approach was not found universally applicable as single trypanosomatid species have been shown to infect more host species and vice versa (Bulat et al., 1999; Podlipaev, 2003; Podlipaev et al., 2004a; Svobodová et al., 2007). Recently, the most promising approach for species description seems to be a combination of several methods including molecular phylogenetics (Batistoti et al., 2001; Yurchenko et al., 2006; Svobodová et al., 2007).

Here, based on morphology, ultrastructure, phylogenetic analysis of SSU rRNA and glycosomal glyceraldehyde phosphate dehydrogenase (gGAPDH) genes, and randomly amplified polymorphic DNA (RAPD) analysis, we describe Herpetomonas trimorpha sp. nov. as a parasite of the intestinal tract of biting midges (Ceratopogonidae), closely related to Herpetomonas ztiplika. The main feature of the novel species is the ability to form three distinct morphotypes in culture. 


\section{METHODS}

Parasites and their cultivation. Females of biting midges Culicoides (Oecacta) truncorum Edwards 1939 (syn. C. sylvarum Callot \& Kremer 1961) were collected into miniature CDC traps when attacking buzzard (Buteo buteo) nestlings in Milovický forest as described previously (Svobodová et al., 2007). The midges were dissected and examined for the presence of parasites. H. trimorpha sp. nov. isolate CER9 was isolated from the Malpighian tubules of a single female. An axenic culture was established by using SNB-9 agar mixed with rabbit blood overlaid with SNB-9 medium containing $10 \%$ fetal calf serum (Sigma), $100 \mu$ g gentamicin $\mathrm{ml}^{-1}, 1000 \mathrm{U}$ penicillin $\mathrm{ml}^{-1}$ and $1.5 \mathrm{mg}$ fluorocytosine $\mathrm{ml}^{-1}$ at room temperature. The medium used for maintaining the culture consisted of RPMI 1640 with HEPES and Schneider's Drosophila medium $(1: 1)$ with $2 \%$ sterile human urine and $25 \mu \mathrm{g}$ gentamicin $\mathrm{ml}^{-1}$. The culture is deposited in the culture collection of the Department of Parasitology, Faculty of Science, Charles University in Prague, Czech Republic, and in the collection of the Academy of Sciences of the Czech Republic, Biology Centre, Institute of Parasitology, České Budějovice, Czech Republic, as strain ICUL/CZ/2001/CER9.

Culture cloning. Exponential phase culture was diluted with fresh medium to 1.3 cells $\mathrm{ml}^{-1}$ and pipetted into three 96-well plates (150 $\mu \mathrm{l}$ per well). Using an inverted microscope, the plates were screened for the presence of cells and their morphotypes for 30 days at different intervals.

Light microscopy. Light-microscopic preparations were made both from cultured cells and from cells extracted from the host during its dissection. The material was smeared on microscopic slides, dried, fixed with methanol, and stained with Giemsa (Sigma). The cell length and width, and the flagellum length of each morphological type ( $n=10$ when available), were measured with a calibrated micrometer (magnification $\times 1000)$. H. trimorpha cells were compared with those of H. ztiplika and Sergeia podlipaevi, the only other trypanosomatids known from biting midges, using ANOVA post-hoc comparisons (Statistica software).

Transmission electron microscopy. Ultrathin electron-microscopic preparations were made from 7-day-old culture in the exponential phase. Cells were washed in $0.1 \mathrm{M}$ PBS solution and fixed in $2.5 \%$ glutaraldehyde in $0.2 \mathrm{M}$ cacodylate buffer at $4{ }^{\circ} \mathrm{C}$ for $24 \mathrm{~h}$, and then post-fixed with $2 \%$ osmium tetroxide in the same buffer at room temperature for $2 \mathrm{~h}$. After dehydration in a graded series of ethanol, cells were embedded in Epon-Araldite. Ultra-thin sections were stained with lead citrate and uranyl acetate, and examined with a JEOL 1011 transmission electron microscope. The length and width of the kinetoplast of 52 specimens were measured from digital photographs by using Grmeasure software. Lengths and widths were processed by ANOVA post-hoc comparisons using Statistica software.

DNA isolation, amplification, cloning and sequencing. Genomic DNA of isolates CER9 of $H$. trimorpha sp. nov. and CER1 of H. ztiplika was extracted with the DNA tissue isolation kit (Roche). The SSU rRNA gene of isolate CER9 was amplified by using primers MedlinA (CTGGTTGATCCTGCCAG) and MedlinB (TGATCCTTCTGCAGGTTCACCTAC) (Medlin et al., 1988). The gGAPDH gene of isolates CER1 and CER9 was amplified by using primers G3 (TTYGCCGYATYGGYCGCATGG) and G4a (GTTYTGCAGSGTCGCCTTGG) (Hamilton et al., 2004). The PCR products were purified and directly sequenced. Additionally, the SSU rRNA gene of isolate CER9 was cloned into the pGEM-T EASY vector (Promega). Nine clones originating from two independent PCRs were partially sequenced using primers T7 (AATACGACTCACTATAG) and SP6 (ATTTAGGTGACACTATAG).
Phylogenetic analyses. Four datasets were created. The first dataset contained 50 SSU rRNA gene sequences of trypanosomatids, including $H$. trimorpha sp. nov., and six sequences of bodonids, which were used as outgroups. The other three datasets contained sequences of the gGAPDH gene. The second dataset contained 61 sequences of various trypanosomatids including newly determined sequences of $H$. trimorpha sp. nov. and H. ztiplika, four bodonid sequences and one euglenid sequence. The bodonid and euglenid gGAPDH sequences were used as outgroups. The third dataset contained 61 trypanosomatid sequences and three outgroup sequences (two of Bodo saltans and one of Parabodo caudatus). The fourth dataset contained only the 61 trypanosomatid gGAPDH sequences.

The sequences were aligned by using CLUSTAL X 1.81 (Thompson et al., 1997) and the resulting alignments were edited manually using BioEdit (Hall, 1999; alignments are available from the corresponding author upon request). Phylogenetic trees were reconstructed by using the maximum-parsimony method, Fitch-Margoliash distance method with maximum-likelihood distances, maximum-likelihood method and Bayesian method. The distance, maximum-parsimony and maximum-likelihood trees were reconstructed in PAUP* $4.0 \mathrm{~b} 10$ (Swofford, 2002) using ten replicates of heuristic search. The starting tree was obtained by the stepwise addition procedure with a random order of taxa addition and swapped using the tree bisectionreconnection algorithm. The models of nucleotide substitution for maximum-likelihood and distance analyses, i.e. $\operatorname{TrNef}+\mathrm{I}+\Gamma$ for the first dataset and GTR $+\mathrm{I}+\Gamma$ for the other three datasets, were chosen by hierarchical nested likelihood ratio tests implemented in modeltest 3.06 (Posada \& Crandall, 1998). The maximum-parsimony and distance trees were bootstrapped in PAUP* with 1000 replicates, each with ten replicates of random taxa addition with TBR branch swapping. Maximum-likelihood trees were bootstrapped in PHYML (Guindon \& Gascuel, 2003) with 1000 replicates. Bayesian analyses were performed using MrBayes 3.1.1 (Ronquist \& Huelsenbeck, 2003). Base frequencies, rates for six different types of substitution, proportion of invariant sites and shape parameter of the gamma correction for the rate heterogeneity with four discrete categories were allowed to vary. The covarion model was used to allow the rate heterogeneity along the tree. The Markov chain Monte Carlo was run for $5 \times 10^{6}$ (first dataset), $2 \times 10^{6}$ (second dataset), $9 \times 10^{6}$ (third dataset), and $8 \times 10^{6}$ (fourth dataset) generations, until the mean standard deviation of split frequencies was lower than 0.01; the trees were sampled every 100th generation. The first 12500 (first dataset), 5500 (second dataset), 22500 (third dataset), and 20000 (fourth dataset) trees were discarded as burn-in. Additionally, the three gGAPDH datasets were translated into amino acids and were subjected to maximum-likelihood analysis implemented in the online version of Bootstrap RAxML (Stamatakis et al., 2008) available on the CIPRES Portal (http://www.phylo.org/sub_sections/portal.php). The following parameters were applied: JTT substitution matrix, empirical base frequencies, maximum-likelihood search, estimate proportion of invariable sites, and 1000 bootstrapping runs.

RAPD analysis. RAPD analysis was performed with eight isolates of three trypanosomatid species: $H$. trimorpha sp. nov. (isolate CER9), $H$. ztiplika (isolates CER1, 2, 6, and 8) and S. podlipaevi (isolates CER3, 4, and 7) (for information on the isolates see Podlipaev et al., 2004b; Svobodová et al., 2007). Twelve primers (Operon technologies) were chosen for the analysis: OPA-03 (AGTCAGCCAC), OPA-09 (GGGTAACGCC), OPA-10 (GTGATCGCAG), OPA-12 (TCGGCGATAG), OPD-03 (GTCGCCGTCA), OPD-08 (GTGTGCCCCA), OPD-11 (AGCGCCATTG), OPD-13 (GGGGTGACGA), OPD-18 (GAGAGCCAAC), OPF-01 (ACGGATCCTG), OPF-05 (CCGAATTCCC) and OPF-14 (TGCTGCAGGT). RAPD products were separated by electrophoresis in $2 \%$ agarose gels. The gels were manually transformed into the matrix: 1 coded the presence and 0 the absence of a character (band of particular size). The matrix was then processed using FreeTree 0.9.1.50 (Pavlíček 
et al., 1999) with the neighbour-joining algorithm (Nei-Li/Dice distances).

Experimental infections. Males and females of biting midges Culicoides (Monoculicoides) nubeculosus were kindly provided by Eric Denison and Karin Darpel (Department of Arbovirology, IAHPirbright laboratory, UK). Three-day-old adults were infected in a batch of 300-400 for $90 \mathrm{~min}$ at $37^{\circ} \mathrm{C}$ on a cotton pad saturated with $15 \%$ sucrose with a 6-day-old culture of $1 \times 10^{6}$ parasites $\mathrm{ml}^{-1}$ in sterile saline solution (Svobodová et al., 2007). The midges were maintained in $250 \mathrm{ml}$ unwaxed paper cups in $100 \%$ relative humidity and were supplemented with $15 \%$ sucrose solution. The temperature was $23{ }^{\circ} \mathrm{C}\left(1-2\right.$ days post-infection, days p.i.) and then $18{ }^{\circ} \mathrm{C}(3-20$ days p.i.). The midges were serially dissected at 1, 3, 5 and 9 days p.i. and examined under a light microscope. The location and density of flagellates in the gut and rectal ampulla were examined.

\section{RESULTS}

Herpetomonas trimorpha sp. nov. was isolated from Malpighian tubules of a biting midge Culicoides truncorum (Ceratopogonidae, Diptera). It has been successfully cultivated from only a single specimen, out of 2500 biting midges of different species examined.

A single morphological cell type was found in the natural host while three distinct morphotypes (microflagellate promastigote, small promastigote and long promastigote) appeared in culture. To test the possibility of transformation to opisthomastigotes, the standard $\left(23^{\circ} \mathrm{C}\right)$ cultivation temperature was increased as described by Roitman et al. (1976). However, no posterior kinetoplast forms were observed, even after $72 \mathrm{~h}$ cultivation at $26{ }^{\circ} \mathrm{C}$. The form found in the host (vector form) was similar to the vector forms of two species previously isolated from biting midges (Herpetomonas ztiplika and Sergeia podlipaevi), and no statistical differences between vector forms of all three trypanosomatid species were found (data not shown). The vector form of $H$. trimorpha sp. nov. was similar to the small promastigote (data not shown).

Cells of the microflagellate promastigote (Fig. 1a and h) had a very short flagellum and a rounded anterior end while the posterior end was pointed. The cells were relatively long and wide with the nucleus and the kinetoplast situated at the anterior end. The microflagellate promastigotes usually showed a tendency to form rosettes, whilst separate cells were observed rarely. The small promastigote cells (Fig. 1b and i) were oval with a flagellum almost two times longer than the cell body. Both the nucleus and the kinetoplast were situated approximately in the middle of the cell. The long promastigote cells (Fig. 1c and j)

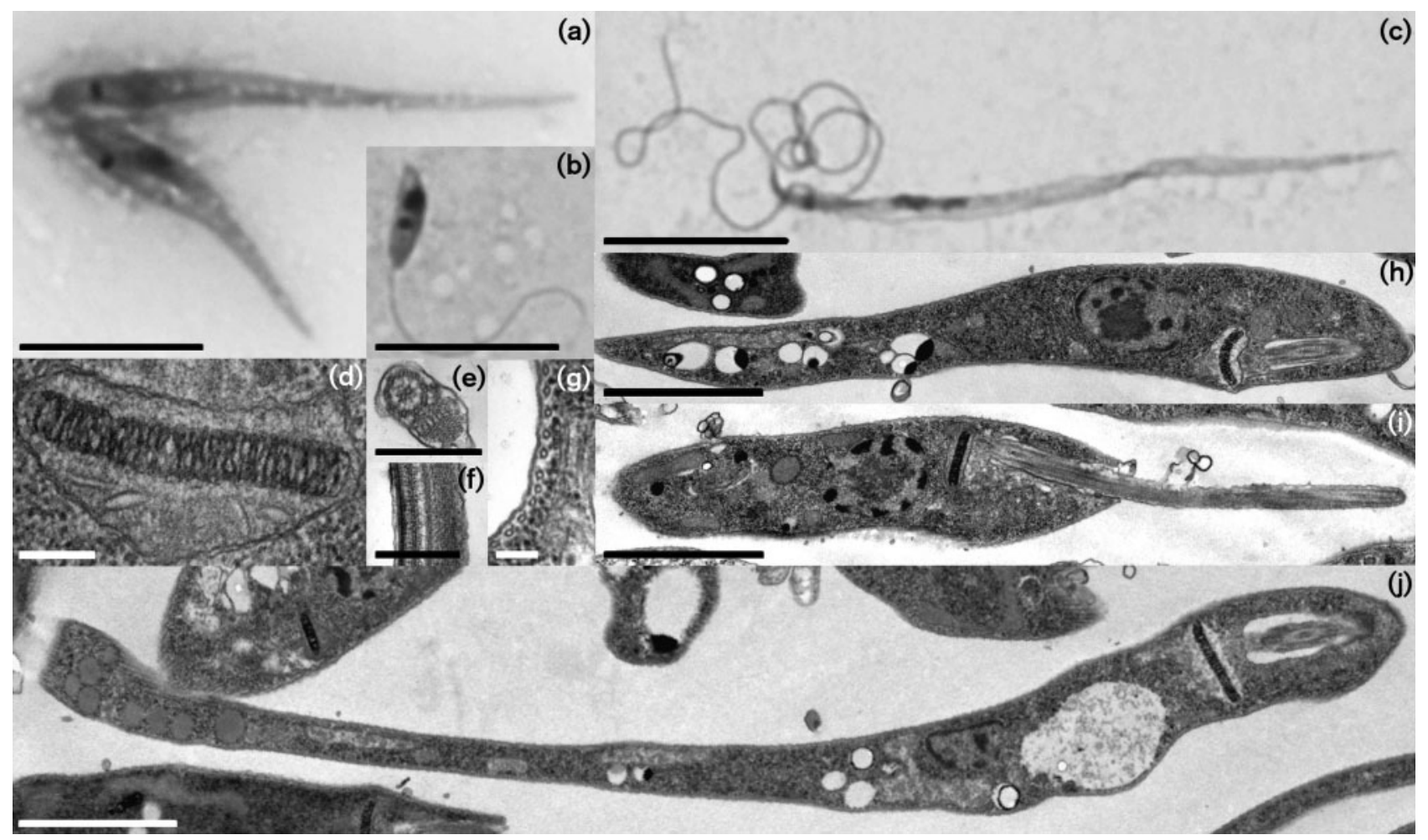

Fig. 1. Light $(a-c)$ and electron $(d-j)$ microscopy of the three morphological types of $H$. trimorpha sp. nov. from culture: microflagellate promastigote (a), small promastigote (b), long promastigote (c), detail of kinetoplast (d) flagellum with a paraflagellar rod in cross (e) and longitudinal ( $f$ ) section, a cross section of a promastigote showing regular spacing of subpellicular microtubules $(\mathrm{g})$, longitudinal sections of a microflagellate promastigote $(\mathrm{h})$, small promastigote (i) and long promastigote (j). Bars, $10 \mu \mathrm{m}(\mathrm{a}-\mathrm{c}), 200 \mathrm{~nm}(\mathrm{~d}), 400 \mathrm{~nm}$ (e and f), $100 \mathrm{~nm}(\mathrm{~g})$ and $2 \mu \mathrm{m}(\mathrm{h}-\mathrm{j})$. 
were extremely long and narrow with both ends pointed. The flagellum was about the same length as the cell. The nucleus was situated in the middle of the cell while the kinetoplast was usually situated in the anterior end of the cell. After transfer to fresh medium, small and microflagellate promastigotes always occurred in culture at the same time while long promastigotes appeared after a short delay (2-5 days). However, all three morphotypes were always present simultaneously in the culture after 5 days.

We compared the body lengths of the three culture forms of $H$. trimorpha sp. nov. to the culture forms of other trypanosomatids obtained from biting midges $(H$. ztiplika and S. podlipaevi) using ANOVA post-hoc comparisons (data not shown). The microflagellate promastigotes of $H$. trimorpha sp. nov. resembled $H$. ztiplika, while the small promastigotes of $H$. trimorpha sp. nov. were similar to the cells of $S$. podlipaevi. The long promastigotes were unique for $H$. trimorpha sp. nov.

Next, $H$. trimorpha sp. nov. was examined by transmission electron microscopy (Fig. 1d-j). The plasmalemma was underlain by parallel subpellicular microtubules with regular spacing (Fig. 1g). The flagellum was supported along its whole length by a paraflagellar rod (Fig. 1e and f). The kinetoplast thickness was $0.125 \pm 0.025 \mu \mathrm{m}$ (Fig. 1d). The size of the minicircle $\mathrm{kDNA}$ was $0.4 \pm 0.08 \mathrm{~kb}$, estimated according to Lukeš \& Votýpka (2000). The proportions of the kinetoplast of $H$. trimorpha sp. nov. were compared with those of $H$. ztiplika. No statistically significant differences were found (data not shown). No endosymbionts were observed inside the parasite cells, which is in agreement with Gadelha et al. (2005) who suggested that the paraflagellar rod of symbiont-bearing trypanosomatid species is always reduced. Additionally, the observed regular spacing of subpellicular microtubules (Fig. 1f) is also typical for endosymbiont-free trypanosomatids (Freymuller \& Camargo, 1981).

A phylogenetic tree based on SSU rRNA gene sequences from the first dataset is shown in Fig. 2. Trypanosomatids formed a robust clade (bootstrap values 83-97, Bayesian posterior probability 1) and split into 11 well-supported lineages whose interrelationships were mostly unresolved and method-dependent. The unresolved backbone of the trypanosomatid SSU rRNA gene sequence tree corresponded to the results of previous analyses (Maslov et al., 2001; Merzlyak et al., 2001; Hughes \& Piontkivska, 2003; Podlipaev et al., 2004a; Svobodová et al., 2007). The genus Trypanosoma (clades I and II) appeared paraphyletic at the base of the trypanosomatid subtree. The paraphyly of the genus Trypanosoma in SSU rRNA gene sequence trees has been reported several times (Vickerman, 1994; Maslov \& Simpson, 1995; Maslov et al., 1996; see Stevens \& Gibson, 1999 for review). A monophyletic genus Trypanosoma was recovered by gGAPDH analyses (see later). Two sequences ascribed to Crithidia oncopelti branched in different positions within lineage VI, indicating that at least one of them was designated erroneously.

In all analyses, the closest relative of $H$. trimorpha sp. nov. was $H$. ztiplika. Both species, together with Herpetomonas pessoai, H. muscarum and H. megaseliae, formed clade IX (bootstrap values 69-99, Bayesian posterior probability 1). Genetic distance (uncorrected $P$ distance) between $H$. trimorpha and $\mathrm{H}$. ztiplika was $0.4 \%$, which is comparable with the distances between some other trypanosomatid species, e.g. H. muscarum L18872 and H. megaseliae.

A phylogenetic tree based on gGAPDH gene sequences from the second dataset is shown in Fig. 3(a). Trypanosomatids formed a robust clade (bootstrap values 80-95, Bayesian posterior probability 1). Similar to the SSU rRNA gene sequence analysis, the backbone of the trypanosomatid clade was poorly resolved. Lineages III (Blastocrithidia triatomae) and X (Herpetomonas mariadeanei) were not represented in the gGAPDH analysis due to the lack of sequences. The genus Trypanosoma (lineages I and II) formed a well-supported clade in the gGAPDH analysis. Clade XI (the genus Phytomonas) was always recovered. Leptomonas collosoma (lineage IV) and Sergeia podlipaevi (lineage V) formed individual branches without clear relationships to the other trypanosomatids. Lineage VI was split into two unrelated clades. Contrary to the SSU rRNA gene sequence analysis, Leptomonas sp. Nfm (lineage VIII) branched robustly within lineage VII indicating that the SSU rRNA gene and gGAPDH gene sequences ascribed to Leptomonas sp. Nfm may belong to different organisms.

Interestingly, lineage IX containing $H$. trimorpha sp. nov. and H. ztiplika was split into three paraphyletic branches at the base of the trypanosomatid subtree. The most basal and at the same time the longest branch was formed by $H$. trimorpha and H. ztiplika. The nucleotide distance between the two species was $8 \%$, which was considerably higher than distances between many closely related trypanosomatid species.

To reduce possible artificial attraction of the outgroups to the long branch of $H$. trimorpha $+H$. ztiplika, the two outgroups with the longest branches, Euglena gracilis and Trypanoplasma borreli, were removed from the analysis (dataset 3). The $H$. trimorpha $+H$. ztiplika branch remained in the basal position in all analyses except for the distance method (bootstrap values 62-84, Bayesian posterior probability 0.99 ; not shown). In the distance analysis, lineage IX was recovered as a clade, although with poor support (bootstrap value 56). When all outgroups were removed (fourth dataset) and an unrooted phylogenetic tree was recreated, lineage IX formed a robust clade (bootstrap values 88-95, Bayesian posterior probability 1; Fig. 3b). H. trimorpha and H. ztiplika formed the longest branch in the tree.

In addition to analyses of the SSU rRNA and gGAPDH genes, we performed a RAPD analysis with $H$. trimorpha sp. nov., H. ztiplika, and S. podlipaevi. Supplementary Figure S1 


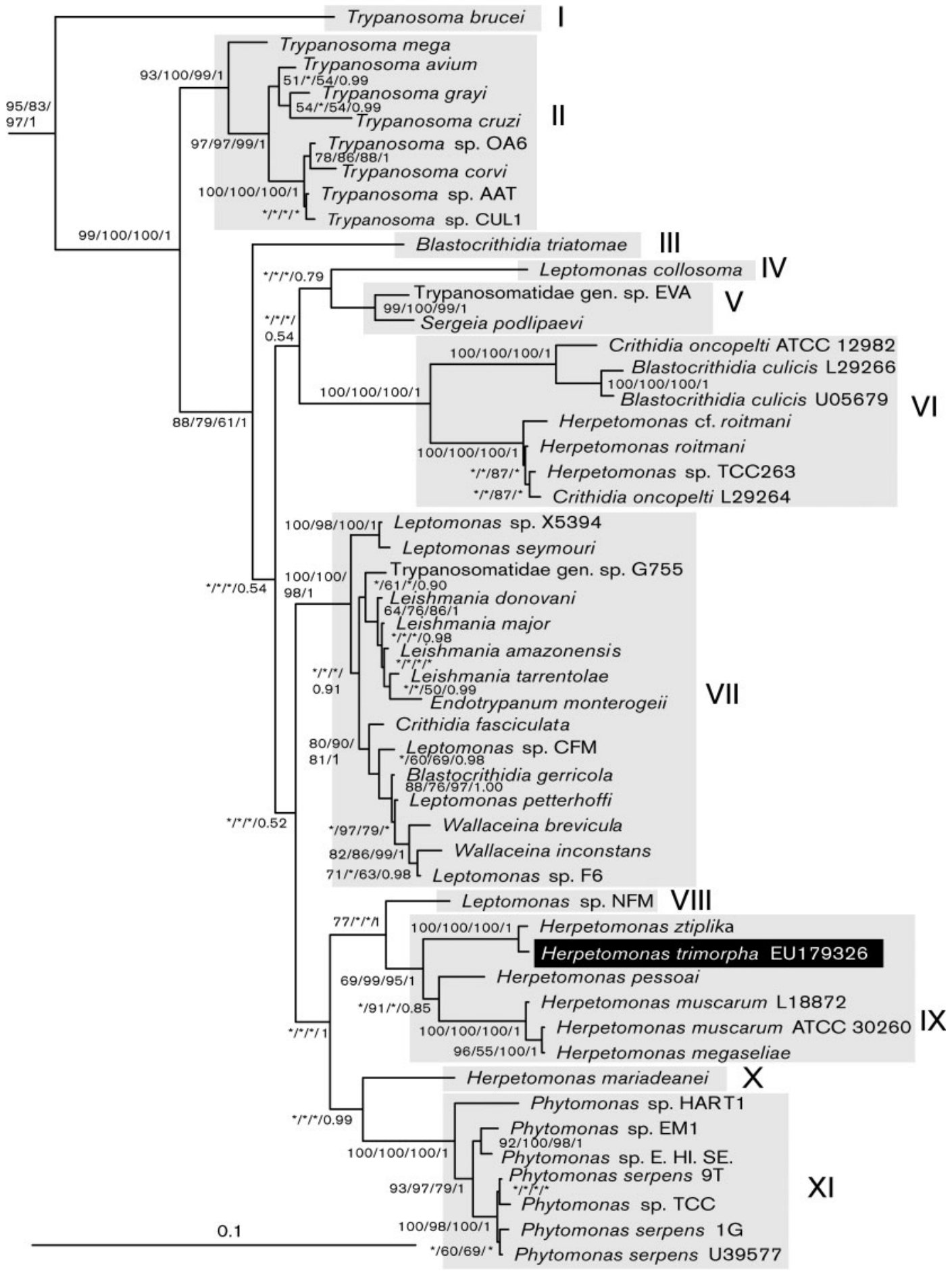

Fig. 2. Phylogenetic tree of trypanosomatids based on SSU rRNA gene sequences and reconstructed by the Bayesian method. Bootstrap values from maximum-parsimony, distance method, maximum-likelihood and Bayesian posterior probabilities are shown at nodes. Asterisks indicate nodes with bootstrap support lower than $50 \%$. The tree was rooted with six bodonid sequences (outgroups not shown). Boxes indicate trypanosomatid lineages. Bar, 0.1 substitutions per site.

(available in IJSEM Online) shows two examples of RAPD gels. The data matrix contained 152 characters. An unrooted dendrogram was reconstructed using the neighbour-joining method (Fig. 4). The genetic distance between $H$. trimorpha and H. ztiplika was comparable to their respective distances to S. podlipaevi. 

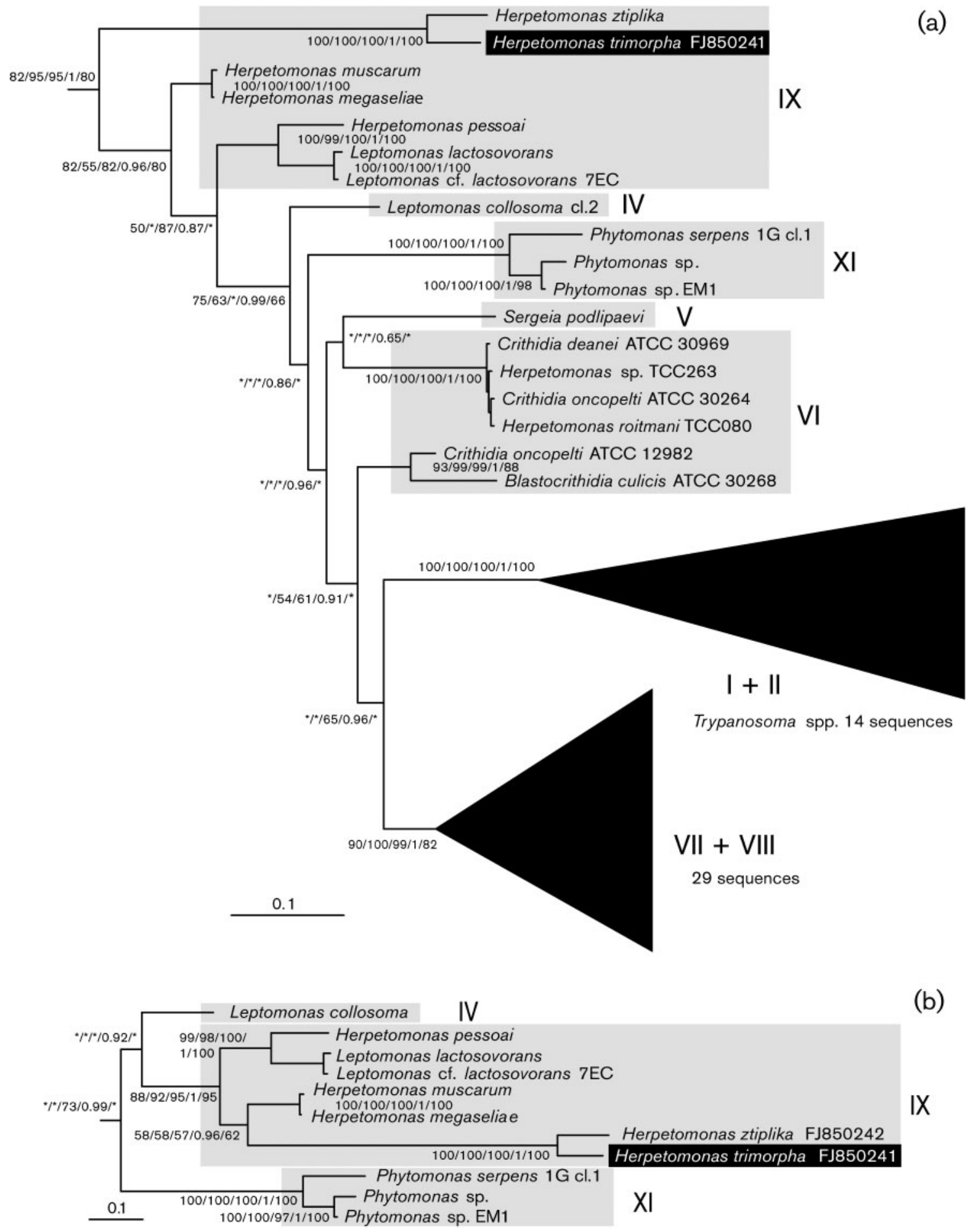

(b)

IX

Fig. 3. Phylogenetic trees of trypanosomatids based on gGAPDH gene sequences. (a) Bayesian tree based on the second dataset. The tree was rooted with five bodonid and euglenid sequences. Bootstrap values from maximum-parsimony, distance method, maximum-likelihood and Bayesian posterior probabilities, and bootstrap values from maximum-likelihood based on amino acids, are shown at nodes. Asterisks indicate nodes with bootstrap support lower than $50 \%$. Boxes indicate trypanosomatid lineages. (b) A part of Bayesian topology when outgroups were removed (dataset 4). Support values for nodes as in Fig. 3a. Bars, 0.1 substitutions per site. 


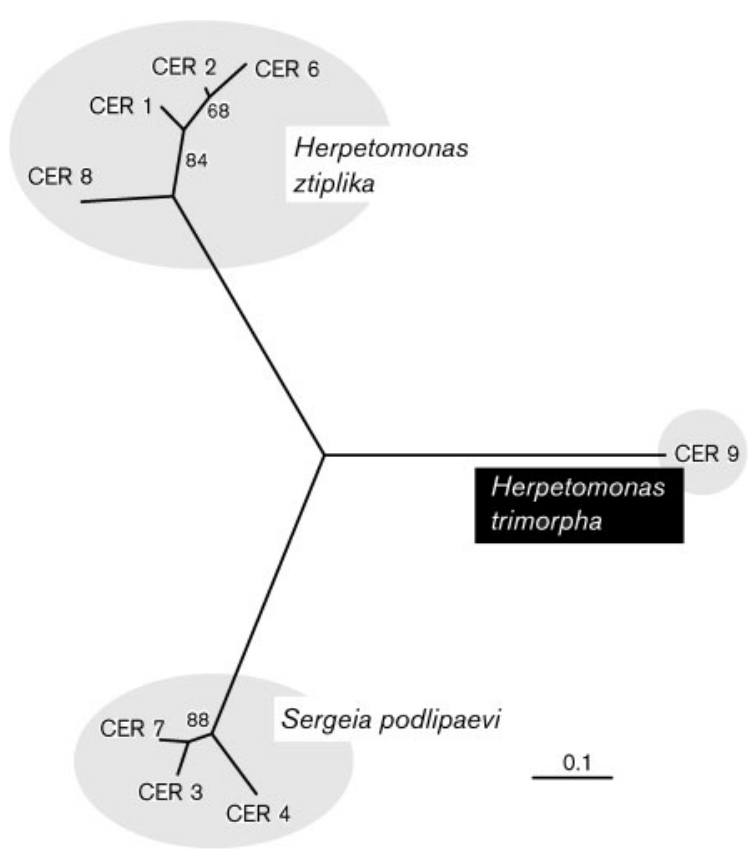

Fig. 4. Unrooted dendrogram based on the dataset of 152 scored RAPD amplicons of Herpetomonas trimorpha sp. nov. (CER9), $\mathrm{H}$. ztiplika (CER1, 2, 6 and 8) and Sergeia podlipaevi (CER3, 4 and 7). Dendrogram reconstructed by using the neighbour-joining method. Bootstrap values are shown at the nodes. Bar, 0.1 substitutions per site.

We employed two independent techniques to examine the conspecificity of the three forms of Herpetomonas trimorpha sp. nov. At first, we analysed the cloned SSU rRNA gene of isolate CER9. At most, two randomly distributed mismatches were observed in nine clones in comparison to the sequence obtained by direct PCR over the $400 \mathrm{bp}$ region analysed. We ascribed the differences to Taq polymerase errors fixed during cloning. There were six mismatches between $H$. trimorpha and $H$. ztiplika over this region. At these six positions, all of the clones had the same sequence as $H$. trimorpha. Later on, we created clonal cell lines derived from isolate CER9. All three cell morphotypes described above occurred in all 13 clonal lines obtained.

We performed an infection experiment with Culicoides nubeculosus using a sugar feeding assay. Only four out of 61 dissected females $(7 \%)$ and one of 37 dissected males (3\%) were found to be infected with $H$. trimorpha. The parasites were found in the midgut, hindgut and Malpighian tubules.

\section{DISCUSSION}

The novel species Herpetomonas trimorpha is the third trypanosomatid described so far from blood-sucking Diptera of the genus Culicoides, the other two species being H. ztiplika (Podlipaev et al., 2004b) and S. podlipaevi (Svobodová et al., 2007). These three species likely represent only a small portion of the real diversity of these parasites in biting midges. H. trimorpha sp. nov. may be a rare species as it was isolated from only one out of 2500 biting midges examined. Nevertheless, we cannot rule out the possibility that $H$. trimorpha is, in fact, common in nature and the low prevalence observed was due either to suboptimal cultivation conditions or to the fact that Culicoides truncorum is only an accidental host and the natural host has not been identified yet. Further attempts to obtain novel $H$. trimorpha isolates from several host taxa are needed to estimate the real abundance of this species in nature.

Both SSU rRNA and gGAPDH gene sequence analyses showed that the closest relative of the novel species is H. ztiplika. Based on the SSU rRNA gene, H. trimorpha sp. nov. and $H$. ztiplika are members of the trypanosomatid clade IX. However, the monophyly of clade IX was not supported in most gGAPDH gene analyses. When the trees were rooted with Euglena gracilis and/or bodonids, clade IX split into three paraphyletic branches at the base of the trypanosomatid subtree, the $H$. trimorpha $+H$. ztiplika branch being the most basal one. When the outgroups were removed, clade IX appeared monophyletic and wellsupported also. The gGAPDH gene of $H$. trimorpha and H. ztiplika apparently underwent an accelerated evolution relative to the other trypanosomatid lineages and to the SSU rRNA gene. We assume that the topologies of gGAPDH trees rooted with Euglena gracilis and/or bodonids obtained may be erroneous due to the long branch attraction artefact, which caused the outgroups to be attracted to the long branch of $H$. trimorpha $H$. ztiplika. Instead of representing the most basal trypanosomatids, the two species are, therefore, most probably closely related to $H$. pessoai, $H$. muscarum, $H$. megaseliae and Leptomonas lactosovorans (SSU rRNA gene sequence of the last species is unavailable).

Three different approaches can be applied to infer the generic assignment of newly isolated trypanosomatid strains. Morphology-based taxonomy follows Wallace (1966), who characterized trypanosomatid genera by morphotypes (e.g. promastigote, opisthomastigote, epimastigote and choanomastigote). Nevertheless, the presence of a certain morphotype is rarely a synapomorphy and most trypanosomatid genera are polyphyletic (Maslov et al., 2001; Merzlyak et al., 2001; Hughes \& Piontkivska, 2003). Application of morphology-based taxonomy also led to the situation where two closely related species belonged to different genera. This is the case of Leptomonas acus and Leptomonas tarcoles, described by Yurchenko et al. (2008), which are closely related to species of the genus Crithidia instead of to other leptomonads. Another possibility is to employ the phylogenetic criterion and to classify a novel strain according to its phylogenetic position. This approach was utilized by Podlipaev et al. (2004b) to describe H. ztiplika. Herpetomonas ztiplika forms only promastigotes and might have been classified as a leptomonad. Nonetheless, it is actually closely related to members of the 
genus Herpetomonas and thus classified as a herpetomonad. Another example is Sergeia podlipaevi described by Svobodová et al. (2007). According to its morphology, S. podlipaevi might also have been classified in the genus Leptomonas. However, as S. podlipaevi is only distantly related to leptomonads, a separate genus was erected for it. Finally, life cycle may serve as an important taxonomic criterion distinguishing trypanosomatid genera as well. This is the case for the morphologically undistinguishable genera Phytomonas and Leptomonas. While leptomonads are monogenetic parasites of insects, phytomonads are digenetic and alternate between insect and plant hosts.

Only promastigotes were observed in strain CER9. The transition to other possible forms may be facilitated by a higher temperature (Faria-e-Silva et al., 1996). Nevertheless, promastigotes remained the only form observed in CER9 when cultured at $26{ }^{\circ} \mathrm{C}$. Therefore, the organism could be assigned to the genera Leptomonas, Phytomonas, Sergeia or Herpetomonas. Since CER9 is not related to the monophyletic or monotypic genera Phytotomonas and Sergeia, it cannot belong to either of them. However, it is difficult to decide whether strain CER9 belongs to the genus Leptomonas or to the genus Herpetomonas. The genus Leptomonas is rather problematic (see Yurchenko et al., 2008). Not only is it highly polyphyletic, but the phylogenetic position of its type species L. bütschlii is unknown. Creating new leptomonad species unrelated to the known ones would, in our opinion, considerably worsen the taxonomical chaos in this genus. In the case of the genus Herpetomonas, almost all species are able to form opisthomastigotes besides promastigotes (Rogers \& Wallace, 1971; Daggett et al., 1972; Roitman et al., 1976; Yoshida et al., 1978; Faria-e-Silva et al., 1996). The only herpetomonad which forms only promastigotes is H. ztiplika (see above). The classification of H. ztiplika in the genus Herpetomonas was broadly accepted (Svobodová et al., 2007; Yurchenko et al., 2009). As the newly described organism is the closest relative of H. ztiplika and is not specifically related to any leptomonad, we decided to assign it to the genus Herpetomonas.

The polyphyletic genus Herpetomonas currently comprises three unrelated trypanosomatid lineages: partial clade VI (H. roitmani), partial clade IX ( $H$. muscarum, $H$. megaseliae, H. pessoai, H. ztiplika and the newly described species) and clade $\mathrm{X}(H$. mariadeanei). The lineages are obviously not congeneric and the genus Herpetomonas has to be divided into at least three genera. However, such taxonomic revision is beyond the scope of the present study. As the type species of the genus Herpetomonas, $H$. muscarum, belongs to the same clade as the new species, $H$. trimorpha sp. nov. represents a 'true' herpetomonad.

It is quite difficult and often impossible to designate morphological characters which would make effective discrimination of trypanosomatid species possible (Yurchenko et al., 2006, 2008, 2009). It is becoming common to characterize a new species solely on the basis of its phylogenetic position rather than its morphology (Podlipaev et al., 2004b; Yurchenko et al., 2006; Svobodová et al., 2007; Yurchenko et al., 2009). Unlike many trypanosomatids, Herpetomonas trimorpha sp. nov. differs considerably in its morphology from its relatives. Above all, it forms three distinct forms: the microflagellate promastigote, the small promastigote and the long promastigote. Although the first two forms have been recorded from $H$. ztiplika and $S$. podlipaevi strains (Podlipaev et al., 2004b; Svobodová et al., 2007), the long promastigote form seems to be unique to H. trimorpha sp. nov. It has not been observed even in the closely related species $H$. ztiplika. Also, the RAPD analysis unambiguously separated $H$. trimorpha sp. nov. from $H$. ztiplika.

Interestingly, Leptomonas lactosovorans, described by Manaia et al. (1981), also belongs to clade IX. It is highly probable that L. lactosovorans is a herpetomonad without the opisthomastigote stage (see Yurchenko et al., 2009), similar to H. ztiplika and H. trimorpha sp. nov. The newly described species differs from $L$. lactosovorans by its long promastigote stage.

Since three different trypanosomatid species have already been isolated from biting midges, the presence of more species in a single isolate was possible. To rule out the possibility of mixed infection in the case of isolate CER9, we employed two independent techniques. At first, we partially sequenced nine clones of the SSU rRNA gene of isolate CER9. Except for a few randomly distributed mismatches, which we ascribed to Taq polymerase errors, the sequences were identical to the SSU rRNA gene sequence originally obtained from strain CER9. Then, we created clonal cell lines derived from isolate CER9. All three morphotypes occurred in all cell lines. Therefore, we concluded that isolate CER9 truly contains only a single species, Herpetomonas trimorpha sp. nov., which forms three different forms in culture. Two or more morphotypes of particular species were also described by other authors (Podlipaev, 1985; Frolov \& Skarlato, 1987; Yurchenko et al., 2006); however, our detailed description of the $H$. trimorpha morphotypes and their continued presence in culture is one of the major contributions of the present study.

It has been suggested that the host is infected by herpetomonads by contaminative infection (McGhee \& Cosgrove, 1980). To test this hypothesis, we performed an infection experiment with Culicoides nubeculosus using a sugar feeding assay. Only $7 \%$ of dissected females and $3 \%$ of dissected males were found to be infected with $H$. trimorpha. The localization of parasites in the laboratory host corresponded to the original findings in its natural hosts. As McGhee \& Cosgrove (1980) proposed, only certain morphological types of trypanosomatids are capable of host infection while others are non-infectious. We suppose this might be true also for $H$. trimorpha sp. nov. Low infection rate could have been caused by differences between the experimental and natural host, which belonged to different species and subgenera. 


\section{TAXONOMIC SUMMARY}

\section{Herpetomonas trimorpha sp. nov.}

Diagnosis. One promastigote morphotype is observed in the host. Cells are oval, $8.2 \pm 0.3(7.5-8.5) \mu \mathrm{m}$ long $(n=3)$ and $2.7 \pm 1.2(2.5-3.0) \mu \mathrm{m}$ wide $(n=3)$; length of flagellum $10.2 \pm 2.3(5.9-13.6) \mu \mathrm{m}(n=3)$. Three distinct promastigote morphotypes are observed in culture (microflagellate promastigote, small promastigote and long promastigote). The cell of the microflagellate promastigote is elongated with a pointed posterior end, $23.8 \pm 1.8(17.0-32.3) \mu \mathrm{m}$ long $(n=10)$ and $3.4 \pm 0.3(2.5-5.9) \mu \mathrm{m}$ wide $(n=10)$; length of flagellum $40.0 \pm 35.9(4.2-76.0) \mu \mathrm{m}(n=2)$. However, the flagellum is usually invisible in Giemsa-stained preparations. The nucleus and the kinetoplast are situated in the anterior end of the cell. The microflagellate promastigotes usually form rosettes. The cell of the small promastigote is oval, $6.2 \pm 0.4(5.1-8.5) \mu \mathrm{m}$ long $(n=10)$ and $2.7 \pm 0.4(1.4-5.2)$ $\mu \mathrm{m}$ wide $(n=10)$; length of flagellum $12.6 \pm 1.0(6.8-17.0) \mu \mathrm{m}$ $(n=10)$. Both the nucleus and the kinetoplast are situated approximately in the middle of the cell. The cell of the long promastigote is long and narrow, $37.9 \pm 0.9(34.0-44.0) \mu \mathrm{m}$ long $(n=10)$ and $1.6 \pm 0.1(1.5-1.7) \mu \mathrm{m}$ wide $(n=10)$; length of flagellum $42.0 \pm 5.8(4.1-68.0) \mu \mathrm{m}(n=10)$. The nucleus is situated in the middle of the cell while the kinetoplast is situated in the anterior end. The thickness of the kinetoplast is $0.125 \pm 0.025 \mu \mathrm{m}$. The flagellum is supported by a paraflagellar rod. No endosymbionts are present in the cells.

Type host. Female of biting midge Culicoides (Oecacta) truncorum Edwards, 1939.

Localization in host. Malpighian tubules.

Type locality. Milovický forest game preserve, in the vicinity of Mikulov (Břeclav district, Southern Moravia, Czech Republic, $48^{\circ} 48^{\prime} 39^{\prime \prime}$ N $16^{\circ} 43^{\prime} 26^{\prime \prime}$ E).

Type isolate. CER9, deposited as ICUL/CZ/2001/CER9 in the collection of the Department of Parasitology, Faculty of Science, Charles University in Prague, Prague, Czech Republic, and in the collection of the Academy of Sciences, Biology Centre, Institute of Parasitology, Ceské Budějovice, Czech Republic.

Syntype slides. Giemsa-stained slides of isolate CER9, both insect and culture forms, deposited in the collection of the Department of Parasitology, Faculty of Science, Charles University in Prague, Prague, Czech Republic.

Etymology Herpetomonas trimorpha (tri.mor'pha. L. tris three, Gr. n. morphê form, shape; N.L. fem. adj. trimorpha which has three forms).

\section{ACKNOWLEDGEMENTS}

We thank Eric Denison and Karin Darpel, Department of Arbovirology, IAH-Pirbright laboratory, UK for provision of the Culicoides nubeculosus midges. We would like to express our thanks to Jiř́ Zídek for programming the Grmeasure software. We also wish to thank Drs Vladimír Hampl, Pavel Doležal and Matthew Rogers for the thorough English review and for the useful comments on the manuscript. This research was supported by the Ministry of Education of the Czech Republic (projects LC06009 and MSM 0021620828).

\section{REFERENCES}

Batistoti, M., Cavazzana, M., Jr, Serrano, M. G., Ogatta, S. F., Baccan, G. C., Jankevicius, J. V., Teixeira, M. M. G. \& Itow Jankevicius, S. (2001). Genetic variability of trypanosomatids isolated from phytophagous hemiptera defined by morphological, biochemical, and molecular taxonomic markers. J Parasitol 87, 1335-1341.

Bulat, S. A., Mokrousov, I. V. \& Podlipaev, S. A. (1999). Classification of Trypanosomatids from insects and plants by the UP-PCR (universally primed PCR) technique and cross dot blot hybridization of PCR products. Eur J Protistol 35, 319-326.

Camargo, E. P., Sbravate, C., Teixeira, M. M. G., Uliana, S. R. B., Soares, M. B. M., Affonso, H. T. \& Floeter-Winter, L. (1992). Ribosomal DNA restriction analysis and synthetic oligonucleotide probing in the identification of genera of lower trypanosomatids. J Parasitol 78, 40-48.

Catarino, L. M., Serrano, M. G., Cavazzana, M., Jr, Almeida, M. L., Kaneshina, E. K., Campaner, M., Jankevicius, J. V., Teixeira, M. M. G. \& Itow-Jankevicius, S. (2001). Classification of trypanosomatids from fruits and seeds using morphological, biochemical and molecular markers revealed several genera among fruit isolates. FEMS Microbiol Lett 201, 65-72.

Daggett, P. M., Dollahon, N. \& Janovy, J., Jr (1972). Herpetomonas megaseliae sp. n. (Protozoa: trypanosomatidae) from Megaselia scalaris (Loew, 1866) Schmitz, 1929 (Diptera: Phoridae). J Parasitol 58, 946949.

Faria-e-Silva, P. M., Soares, M. J. \& De Souza, W. (1996). Proliferative opisthomastigote forms in Herpetomonas roitmani (Kinetoplastida: Trypanosomatidae). Parasitol Res 82, 125-129.

Fiorini, J. E., Takata, C. S. A., Teofilo, V. M., Nascimento, L. C., Faria-e-Silva, P. M., Soares, M. J., Teixeira, M. M. G. \& De Souza, W. (2001). Morphological, biochemical and molecular characterization of Herpetomonas samuelpessoai camargoi n. subsp., a trypanosomatid isolated from the flower of the squash Cucurbita moschata. J Eukaryot Microbiol 48, 62-69.

Freymuller, E. \& Camargo, E. P. (1981). Ultrastructural differences between species of trypanosomatids with and without endosymbionts. J Protozool 28, 175-182.

Frolov, A. O. \& Skarlato, S. O. (1987). Light- and electron microscopical study of Leptomonas pyrrhocoris Z. (Kinetoplastida, Trypanosomatidae). Parazitologiia 21, 3-9 (in Russian with English summary).

Gadelha, C., Wickstead, B., de Souza, W., Gull, K. \& Cunha-e-Silva, N. (2005). Cryptic paraflagellar rod in endosymbiont-containing kinetoplastid protozoa. Eukaryot Cell 4, 516-525.

Guindon, S. \& Gascuel, O. (2003). A simple, fast, and accurate algorithm to estimate large phylogenies by maximum likelihood. Syst Biol 52, 696-704.

Hall, T. A. (1999). BioEdit: a user-friendly biological sequence alignment editor and analysis program for Windows 95/98/NT. Nucleic Acids Symp Ser 41, 95-98.

Hamilton, P. B., Stevens, J. R., Gaunt, M. W., Gidley, J. \& Gibson, W. C. (2004). Trypanosomes are monophyletic: evidence from genes for glyceraldehyde phosphate dehydrogenase and small subunit ribosomal RNA. Int J Parasitol 34, 1393-1404. 
Hollar, L., Lukeš, J. \& Maslov, D. A. (1998). Monophyly of endosymbiont containing trypanosomatids: phylogeny versus taxonomy. J Eukaryot Microbiol 45, 293-297.

Hughes, A. L. \& Piontkivska, H. (2003). Phylogeny of Trypanosomatidae and Bodonidae (Kinetoplastida) based on $18 \mathrm{~S}$ rRNA: evidence for paraphyly of Trypanosoma and six other genera. Mol Biol Evol 20, 644-652.

Lukeš, J. \& Votýpka, J. (2000). Trypanosoma avium: novel features of the kinetoplast structure. Exp Parasitol 96, 178-181.

Manaia, A. C., Souza, M. C. M., Lustosa, É. S. \& Roitman, I. (1981). Leptomonas lactosovorans $\mathrm{n}$. sp., a lactose-utilizing trypanosomatid: description and nutritional requirements. J Eukaryot Microbiol 28 , 124-126.

Marín, C., Fabre, S., Sánchez-Moreno, M. \& Dollet, M. (2007). Herpetomonas spp. isolated from tomato fruits (Lycopersicon esculentum) in southern Spain. Exp Parasitol 116, 88-90.

Maslov, D. A. \& Simpson, L. (1995). Evolution of parasitism in kinetoplastid protozoa. Parasitol Today 11, 30-32.

Maslov, D. A., Lukeš, J., Jirků, M. \& Simpson, L. (1996). Phylogeny of trypanosomes as inferred from the small and large subunit rRNAs: implications for the evolution of parasitism in the trypanosomatid protozoa. Mol Biochem Parasitol 75, 197-205.

Maslov, D. A., Podlipaev, S. A. \& Lukeš, J. (2001). Phylogeny of the Kinetoplastida: taxonomic problems and insights into the evolution of parasitism. Mem Inst Oswaldo Cruz 96, 397-402.

McGhee, R. B. \& Cosgrove, W. B. (1980). Biology and physiology of the lower Trypanosomatidae. Microbiol Rev 44, 140-173.

Medlin, L., Elwood, H. J., Stickel, S. \& Sogin, M. L. (1988). The characterization of enzymatically amplified eukaryotic 16S-like rRNA-coding regions. Gene 71, 491-499.

Merzlyak, E., Yurchenko, V., Kolesnikov, A. A., Alexandrov, K., Podlipaev, S. A. \& Maslov, D. A. (2001). Diversity and phylogeny of insect trypanosomatids based on small subunit rRNA genes: polyphyly of Leptomonas and Blastocrithidia. J Eukaryot Microbiol 48, 161-169.

Molyneux, D. (1977). Vector relationships in the Trypanosomatidae. Adv Parasitol 15, 54-58.

Momen, H. (2001). Some current problems in the systematics of trypanosomatids. Int J Parasitol 31, 640-642.

Morsy, T. A., Schnur, L. F., Feinsod, F. M., Michael, S. A., Saah, A., Salma, M. M. \& Wahba, M. M. (1988). The discovery and preliminary characterization of a novel trypanosomatid parasite from Rattus norvegicus and stray dogs from Alexandria, Egypt. Ann Trop Med Parasitol 82, 437-444.

Nunes, L. R., Teixeira, M. M. G., Camargo, E. P. \& Affonso, H. T. (1994). kDNA and rDNA sequences reveal a phylogenetic cluster of species originally placed in different genera of trypanosomatids. J Eukaryot Microbiol 41, 496-500.

Pavlíček, A., Hrdá, Š. \& Flegr, J. (1999). FreeTree - freeware program for construction of phylogenetic trees on the basis of distance data and bootstrap/jackknife analysis of the tree robustness. Application in the RAPD analysis of the genus Frenkelia. Folia Biol (Praha) 45, 97-99.

Podlipaev, S. A. (1985). New species of lower trypanosomatids from Heteroptera families Gerridae and Nabidae: stages of their life cycles in nature and in the laboratory. Proc Zool Inst Leningrad 129, 35-47 (in Russian with English summary).

Podlipaev, S. A. (1990). Catalogue of world fauna of Trypanosomatidae (Protozoa). Proc Zool Inst Leningrad 144, 1-178.

Podlipaev, S. A. (2000). Insect trypanosomatids: the need to know more. Mem Inst Oswaldo Cruz 95, 517-522.
Podlipaev, S. A. (2001). The more insect trypanosomatids under study the more diverse Trypanosomatidae appears. Int J Parasitol 31, 648-652.

Podlipaev, S. A. (2003). Host specificity of homoxenous trypanosomatids. Parazitologiia 37, 3-17 (in Russian with English summary).

Podlipaev, S. A., Sturm, N. R., Fiala, I., Fernandes, O., Westenberger, S. J., Dollet, M., Campbell, D. A. \& Lukeš, J. (2004a). Diversity of insect trypanosomatids assessed from the spliced leader RNA and 5S rRNA genes and intergenic regions. J Eukaryot Microbiol 51, 283-290.

Podlipaev, S. A., Votýpka, J., Jirků, M., Svobodová, M. \& Lukeš, J. (2004b). Herpetomonas ztiplika sp. nov. (Kinetoplastida: Trypanosomatidae): a parasite of the blood-sucking biting midge Culicoides kibunensis Tokunaga, 1937 (Diptera: Ceratopogonidae). J Parasitol 90, 342-347.

Posada, D. \& Crandall, K. A. (1998). MODELTEST: testing the model of DNA substitution. Bioinformatics 14, 817-818.

Rogers, W. E. \& Wallace, F. G. (1971). Two new subspecies of Herpetomonas muscarum (Leidy, 1856) Kent, 1880. J Protozool 18, 645-654.

Roitman, I., Brener, Z., Roitman, C. \& Kitajima, E. W. (1976). Demonstration that Leptomonas pessoai Galvão, Oliveira, Carvalho \& Veiga, 1970, is a Herpetomonas. J Protozool 23, 291-293.

Ronquist, F. \& Huelsenbeck, J. P. (2003). MrBayes 3: Bayesian phylogenetic inference under mixed models. Bioinformatics 19, 1572-1574.

Santos, A. L. S., Abreu, C. M., Alviano, C. S. \& Soares, R. M. A. (2005). Use of proteolytic enzymes as an addititional tool for trypanosomatid identification. Parasitology 130, 79-88.

Stamatakis, A., Hoover, P. \& Rougemont, J. (2008). A rapid bootstrap algorithm for the RAxML web servers. Syst Biol 57, 758-771.

Stevens, J. R. \& Gibson, W. (1999). The molecular evolution of trypanosomes. Parasitol Today 15, 432-437.

Svobodová, M., Zídková, L., Čepička, I., Oborník, M., Lukeš, J. \& Votýpka, J. (2007). Sergeia podlipaevi gen. nov., sp. nov. (Trypanosomatidae, Kinetoplastida), a parasite of biting midges (Ceratopogonidae, Diptera). Int J Syst Evol Microbiol 57, 423-432.

Swofford, D. L. (2002). PAUP*: Phylogenetic analysis using parsimony (and other methods), version 4. Sunderland, MA: Sinauer Associates.

Teixeira, M. M. G., Takata, C. S. A., Conchon, I., Campaner, M. \& Camargo, E. P. (1997). Ribosomal and kDNA markers distinguish two subgroups of Herpetomonas among old species and new trypanosomatids isolated from flies. J Parasitol 83, 58-65.

Thompson, J. D., Gibson, T. J., Plewniak, F., Jeanmougin, F. \& Higgins, D. G. (1997). The CLUSTAL_X windows interface: flexible strategies for multiple sequence alignment aided by quality analysis tools. Nucleic Acids Res 25, 4876-4882.

Vickerman, K. (1994). The evolutionary expansion of the trypanosomatid flagellates. Int J Parasitol 24, 1317-1331.

Wallace, F. G. (1966). The trypanosomatid parasites of insects and arachnids. Exp Parasitol 18, 124-193.

Wallace, F. G., Camargo, E. P., McGhee, R. B. \& Roitman, I. (1983). Guidelines for the description of new species of lower trypanosomatids. J Eukaryot Microbiol 30, 308-313.

Yoshida, N., Freymüller, E. \& Wallace, F. G. (1978). Herpetomonas mariadeanei sp. n. (Protozoa, Trypanosomatidae) from Muscina stabulans (Falléen, 1816) (Diptera, Muscidae). J Eukaryot Microbiol 25, 421-425.

Yurchenko, V., Lukeš, J., Xu, X. \& Maslov, D. A. (2006). An integrated morphological and molecular approach to a new species description in the Trypanosomatidae: the case of Leptomonas podlipaevi n. sp., a parasite of Boisea rubrolineata (Hemiptera: Rhopalidae). J Eukaryot Microbiol 53, 103-111. 
Yurchenko, V. Y., Lukeš, J., Tesařová, M., Jirků, M. \& Maslov, D. A. (2008). Morphological discordance of the new trypanosomatid species phylogenetically associated with the genus Crithidia. Protist 159, 99-114.
Yurchenko, V. Y., Lukeš, J., Jirků, M. \& Maslov, D. A. (2009). Selective recovery of the cultivation-prone components from mixed trypanosomatid infections: a case of several novel species isolated from Neotropical Heteroptera. Int J Syst Evol Microbiol 59, 893-909. 